\title{
Coordination Technologies for Managing Information System Evolution
}

\author{
Luís Filipe Andrade ${ }^{1}$ and José Luiz Fiadeiro ${ }^{2}$ \\ ${ }^{1}$ ATX Software S.A. \\ Alameda António Sérgio 7 - 1 A, \\ 2795 Linda-a-Velha, Portugal \\ landrade@oblog.pt \\ 2 LabMAC \& Dept. of Informatics \\ Faculty of Sciences, University of Lisbon \\ Campo Grande, 1700 Lisboa, Portugal \\ jose@fiadeiro.org
}

\begin{abstract}
Information System Engineering has become under increasing pressure to come up with software solutions that endow systems with the agility that is required to evolve in a continually changing business and technological environment. In this paper, we suggest that Software Engineering has a contribution to make in terms of concepts and techniques that have been recently developed for Parallel Program Design and Software Architectures, which we have named Coordination Technologies. We show how such mechanisms can be encapsulated in a new modelling primitive - coordination contract - that can be used for extending Component Based Development approaches in order to manage such levels of change.
\end{abstract}

\section{Introduction}

More and more, companies live in a very volatile and turbulent environment in which both business rules and supporting technology change very quickly. In order to remain competitive, companies need their information systems to be easily adaptable to such changes, most of the time in a way that does not imply interruptions to critical services. Through the advent of the Internet and Wireless Applications, the New Economy is only fuelling this need even further: "... the ability to change is now more important than the ability to create e-commerce systems in the first place. Change becomes a first-class design goal and requires business and technology architecture whose components can be added, modified, replaced and reconfigured" [15].

Component-Based Development (CBD) [28] has been often proclaimed to be the ultimate approach for providing software solutions with the agility required to cope with such turbulence in business and technological environments. As put in [21], "Software developers have long held the belief that complex systems can be built from smaller components, bound together by software that creates the unique behav- 
iour and forms of the system. Ideally, a new system can be built using mostly predefined parts, with only a small number of new components required... In a well designed system, the changes will be localised, and the changes can be made to the system with little or no effect on the remaining components".

However, it is also widely recognised that the promises brought in by CBD are not without several caveats. Our own experience in developing applications in one of the most volatile business areas - banking - indicates that interactions and architectures are at the core of the problems that still need to be addressed before CBD can deliver the goods. Many of the changes required on a system do not concern the computations performed by its components but the way they interact. Indeed, we often forget that the global behaviour of a system emerges both from the local behaviour of its components and the way they are interconnected. In dynamic business areas, the most frequent changes are likely to occur not at the level of the core entities of the domain (say, the notion of a bank account) but at the level of the business rules that determine how these core entities interact (say, how customers interact with their accounts). The old-fashioned technique of coding interactions in the components that implement the core services (say, reflecting policies on cash withdrawals directly on the withdrawal operation of an account) leads to systems that are very difficult to evolve because any change on the business rules triggers changes on the core components that it involves, their clients, possibly their client's clients, and so on.

Notice that object-oriented methods are very old-fashioned in this respect: interactions between objects are usually coded in the way messages are passed, features are called, and objects are composed, leading to intricate spaghetti-like structures that are difficult to understand, let alone change. Hence, new methodological concepts and supporting technology are needed that promote interactions to first-class entities, leading to systems that are "exoskeletal" in the sense that they exhibit their configuration structure explicitly [23]. Such an externalisation of the interconnections should allow for systems to be reconfigured, in a compositional, non-intrusive way, by acting directly on the entities that capture the interactions between components, leading to an evolution process that follows the architecture of the system $[11,26]$.

Whereas interactions relate two or more components, the technology that is required for externalising them is also meaningful when applied to the evolution of single components. Indeed, another class of changes often required on a system concerns the adaptation of individual components [9], either existing ones (as in legacy systems) or newly acquired ones (as from third-party suppliers). In both cases, the implementation of the components is often not available and new ones cannot be brought in to replace them. A solution is to connect each component that needs to be evolved to an adapter such that the behaviour that emerges from the interaction between component and adapter fulfils the changes that were required initially. The advantage of making this interconnection explicit is that the adapter can be evolved independently of the component that it regulates, thus adding flexibility with respect to future changes.

In order to cope with the complexity of system evolution, we need more than mechanisms for managing change: we need to be able to determine what needs to be changed. Because change is most easily perceived and understood at the level of the 
application domain, the component model must be abstract enough to accommodate these changes without bringing in implementation details. This requires that the more traditional low-level notion of software component, and the design techniques used for its development (e.g., design patterns [17]), be "upgraded" to account for entities that are meaningful at the level of the application domain. This reflects the principle of "business centricity" upheld in [20] according to which software should be architected such that it reflects the way business organisations, policies and processes are defined by their own people. The ultimate goal of this approach is "100\% maintenance" by which the authors mean "continuous quick evolution performed directly by the user rather than by the producer of the system".

This "isomorphism" between business component and software component architecture requires that changes perceived at the level of the business domain be mapped to the implementation levels in a compositional, non-intrusive way by keeping them local to the implementations of the higher-level parts that need to be changed. In summary, the architectural structure of the system - its gross decomposition in terms of high-level components and the interconnections that bind them together - should reflect the structure of the business domain, and mechanisms should be provided that enable evolution to be compositional over this structure.

In this paper, we assert that the entities that provide interconnections between components and the adapters of single components, all of which are called coordination contracts in the rest of the paper, should be seen as coordination mechanisms that one may superpose, in a non-intrusive way, on existing systems, supporting "plug-and-play" in system construction and evolution. The terms "coordination" and "superposition" in the previous sentence have a precise technical meaning and they constitute the kernel of the contribution that we think Software Engineering can make to enhancing the agility of information systems.

Through "coordination" we mean principles and techniques that have been recently developed for separating what in systems accounts for the computations that are performed and the interactions that are maintained [18]. Our belief is that CBD needs to be enhanced with primitives that provide for the explicit representation of the coordination mechanisms that regulate the way components behave and interact, and enable systems to evolve through the reconfiguration of the coordination mechanisms in place, without having to interfere with the way the computations performed by the individual components are programmed.

The term "superposition" refers to a mechanism, also called "superimposition" by some authors, that has been developed and applied in Parallel Program Design for extending systems while preserving their properties [7,10,16,22]. Our belief is that this basic mechanism is lacking in object-oriented development approaches and fills the gap that we have identified in $\mathrm{OO}$ development for supporting the externalisation of interactions and their evolution.

These are the two sides of the coin that we call Coordination Technologies [5] and with which we want to contribute to the debate that has been launched for identifying synergies between Software and Information System Engineering. In section 2, we provide further motivation on what these Coordination Technologies are and how they can contribute to increased levels of agility in the next generations of informa- 
tion systems. In section 3, we discuss an extension that we proposed in [4] for the UML in terms of a new semantic primitive that encapsulates Coordination Technology - coordination contracts or, simply, contracts. In section 4 , we discuss the semantics and deployment of contracts. We conclude with an overview of what is available on contracts and what we are planning to make available in the near future.

\section{Motivation}

In order to motivate the principles and techniques that we wish to put forward through Coordination Technologies, consider the familiar world of bank accounts and clients who can make deposits, withdrawals, transfers, and so on. The notation that we will use in the examples is a shortened version of the Oblog language [www.oblog.com] that we have been developing for object-oriented modelling [3]. An example of a class specification is given below for bank accounts.

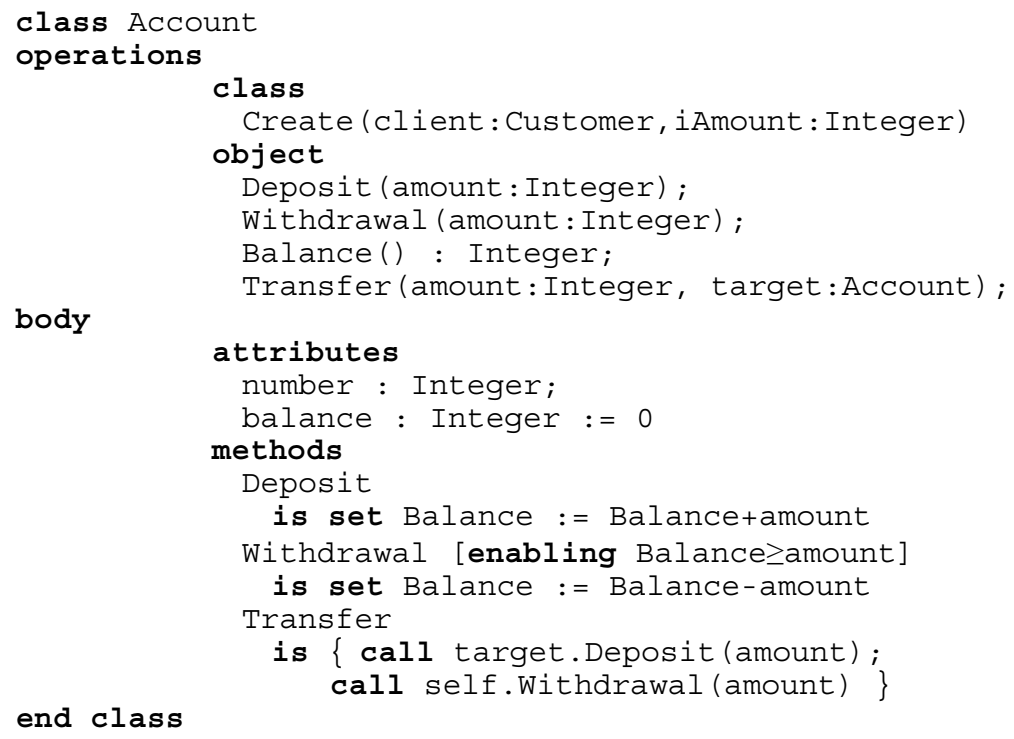

In Oblog, a class specification includes a section in which the interface operations are declared. We distinguish between class and object operations: the former are used for managing the population of the class as a whole and the latter apply to each specific instance. Each operation is declared with a list of input and output parameters and a specification of its required behaviour in terms of pre/post conditions (omitted in the example for simplicity). In the case of the bank account, the operations that were chosen are self-explanatory.

The body section of a class specification identifies the attributes that define the state of the instances as well as the implementations of the operations (called methods). Methods can be guarded with state-conditions like in Dijkstra's guarded com- 
mands. This is the case of withdrawal that is being restricted to occur in states in which the amount to be withdrawn can be covered by the balance.

This restriction on the availability of withdrawals is a good example of the limitations of object-oriented methods for supporting the flexibility that is required for managing change. Indeed, the enabling condition Balance zamount derives more from the specification of a business requirement than an intrinsic constraint on the functionality of a basic business entity like Account. The circumstances under which a withdrawal can be accepted is likely to change as competition dictates banks to come up with new ways for customers to interact with their accounts. Different financial packages are concocted every now and then that provide different policies on withdrawals, making it impossible to predict how accounts will be accessed over their lifetime. Nevertheless, there are basic functional properties that should remain much more stable, reflecting the core "semantics" of the operation as a business transaction, like the fact that the amount is actually deducted from the balance.

One could argue that, through inheritance, this guard could be changed in order to model these different situations and future ones that may arise as a consequence of changes on business policies. However, there are two main problems with the use of inheritance for this purpose. Firstly, inheritance views objects as white boxes in the sense that adaptations like changes to guards are performed on the internal structure of the object. From the point of view of evolution, this is not desirable. This is because, on the one hand, changes on the internal structure are often difficult to locate and localise because they may trigger changes to the interface of the component as well and, thus, initiate a cascade of changes throughout the chain of clientship. On the other hand, inheritance may not be applicable when the component structure is simply not available, as in the case of legacy systems or third-party components.

Secondly, from the business point of view, the adaptations that make sense may be required on classes other than the ones in which the restrictions were implemented. In the example above, this is the case when it is the type of client, and not the type of account, that determines the nature of the guard that applies to withdrawals. The reason the restriction ended up coded as a guard on withdrawals results already from the bias that the $\mathrm{OO}$ mechanism of clientship introduces in the modelling of interactions. Typically, restrictions of this sort are coded up on the server-side, which is where the operation is usually implemented, even if they are meant to reflect access modes that result from different categories of clients.

Hence, it makes more sense for business requirements of this sort to be modelled explicitly outside the classes that model the basic business entities. Our proposal is that guards like the one discussed above should be modelled as coordination contracts that can be established between bank customers and accounts, but outside the specifications of these two classes as explicit representations of the business rules from which they derive. In fact, we provide mechanisms for such contracts to be superposed on existing implementations of clients and accounts, considered as black boxes, so that contracts can be added and deleted in a non-intrusive way (plug and play), reflecting the evolution of the business domain. 


\section{Coordination Contracts}

From a static point of view, a coordination contract defines an association class in the sense of the UML (i.e. an association that has all the attributes of a class). However, the way interaction is established between the partners is more powerful: it provides a coordination role that is closer to what is available for configurable distributed systems in general, namely through the use of architectural connectors [2]. A contract consists of a collection of role classes (the partners in the contract) and the prescription of the coordination effects (the glue in the terminology of software architectures) that will be superposed on the partners. In Oblog, contracts are defined as follows:

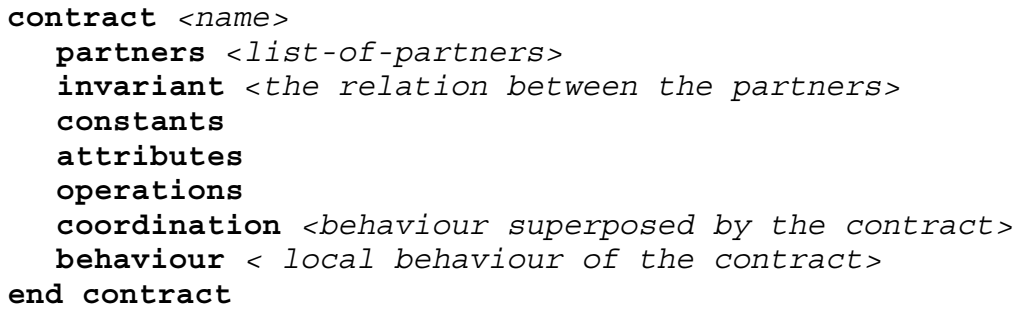

The instances of the partners that can actually become coordinated by instances of the contract are determined through a set of conditions specified as invariants. The typical case is for instances to be required to belong to some association between the partners.

The behaviour that is required to be superposed over that of the partners is identified under "coordination" as trigger/reaction clauses of the form

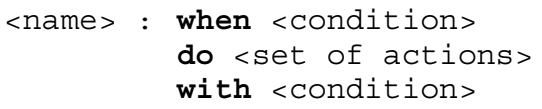

Each coordination clause has a name that can be used for managing the interference between all the clauses and the contract's own actions, e.g. in terms of precedence relations. This is similar to what happens in parallel program design languages like Interacting Processes [16]. The condition under "when" establishes the trigger of the clause. Typical triggers are the occurrence of actions or state changes in the partners. Under "do" we identify the reactions to be performed, usually in terms of actions of the partners and some of the contract's own actions, which constitute what we call the synchronisation set associated with the trigger. Finally, under "with", we can put further constraints on the actions involved in the synchronisation set, typically further preconditions. The intuitive semantics (to be further discussed in the following section) is that, through the "when" condition, the contract intercepts calls to the partners or detects events in the partners to which it has to react. It then checks the "with" conditions to determine whether the interaction can proceed and, if so, coordinates the execution of the synchronisation set. All this is done atomically, in a transactional mode, in the sense that either all the actions in the set are executed or none is.

An example can be given through the account packages already discussed. The traditional package, by which withdrawals require that the balance be greater than the 
amount being withdrawn, can be specified as follows, where by Account we mean the class specification discussed in the previous section but without the enabling restriction on withdrawals:

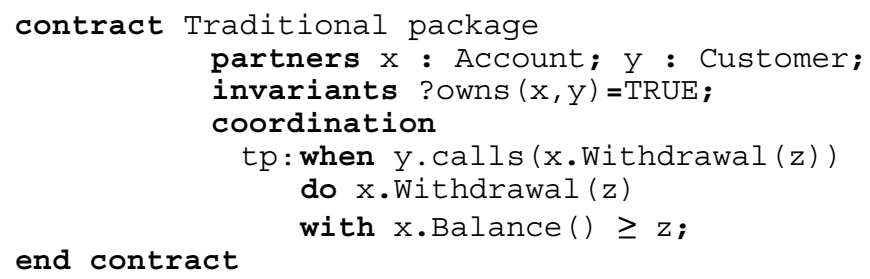

Notice that, as specified by the invariant, this contract is based on an ownership association that must have been previously defined. This contract involves only one interaction. It relates calls placed by the customer for withdrawals with the actual withdrawal operation of the corresponding account. The customer is the trigger of the interaction: the interaction requires every call of the customer to synchronise with the withdrawal operation of the account but enables other withdrawals to occur outside the interactions, e.g. by other joint owners of the same account. The constraint imposed through the with-clause is the guard already discussed in the previous section, which is now externalised as part of the contract. Hence, it regulates only a specific class of interactions between customers and accounts: those that have subscribed to the particular contract Traditional package. In particular, each instance of the contract and, hence, the constraint, applies only to an identified pair of customer and account, meaning that other owners of the same account may subscribe to different contracts.

The notation involving the interaction in this example is somewhat redundant because the fact that the trigger is a call from the customer to an operation of the account immediately identifies the reaction to be performed. In situations like this, Oblog allows for abbreviated syntactical forms. However, in the paper, we will consistently present the full syntax to make explicit the various aspects involved in an interaction. In particular, the full syntax makes it explicit that the call put by the client is intercepted by the contract, and the reaction, which includes the call to the supplier, is coordinated by the contract. Again, we stress that the interactions established through contracts are atomic, i.e. the synchronisation set determined by each coordination entry of the contract is executed as a single transaction - either all the actions in the set are performed or none is. In particular, the client will not know what kind of coordination is being superposed. From the point of view of the client, it is the supplier that is being called.

As already explained, the purpose of contracts is to externalise interactions between objects, making them explicit in the conceptual models, thus reflecting the business rules that apply in the current state. Hence, contracts may change as the business rules change, making system evolution compositional with respect to the evolution of the application domain. For instance, new account packages may be introduced that relax the conditions under which accounts may be overdrawn: 


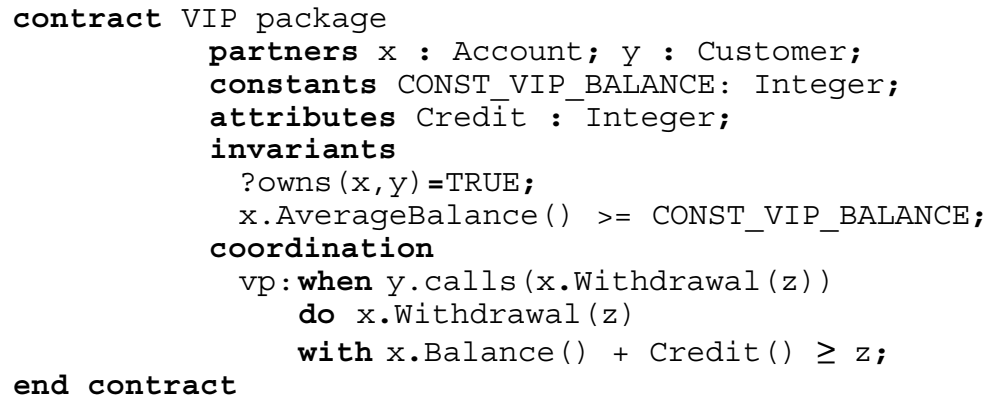

Notice that, on the one hand, we have strengthened the invariant of the contract, meaning that only a restricted subset of the population can become under the coordination of this new business rule, namely the customers that qualify as VIPs. On the other hand, the contract weakens the guard imposed on withdrawals, meaning that there are now more situations in which customers can withdraw money from their accounts: this is the added benefit of the more restricted class of customers.

In general, we allow for contracts to have features of their own. This is the case of the contract above for which an attribute and a constant were declared to model the credit facility. It is important to stress that such features (including any additional operations) are all private to the contract: they fulfil a local purpose in establishing the coordination that is required between the partners and are not available for interaction with other objects. Indeed, the contract does not define a public class and its instances are not considered as ordinary components of the system. This is one of the reasons why association classes, as available in the UML, are not expressive enough to model the coordination mechanisms of contracts. Although contracts allow for interactions to be made explicit in conceptual models, they should not be accessed in the same way as the classes that model the core business entities. Contracts do not provide services: they coordinate the services made available by the core entities. Contracts are the subject of a different level of management of the business component system: that of the definition and evolution of its configuration.

Another shortcoming of association classes is that they do not enforce the synchronisation and atomicity requirements of the proposed coordination mechanisms. As an example, consider one of the latest products to appear in banking - "the flexible package". This is a mechanism via which automatic transfers are made between a checking account and a savings account of the same client: from savings to checking when the balance is not enough to satisfy a withdrawal, and from checking to savings when the balance goes above a certain threshold.

Like before, the application of traditional object-oriented techniques for adding this new feature to the system would probably raise a number of problems. The first one concerns the decision on where to place the code that is going to perform the transfers: the probable choice would be the checking account because that is where the balance is kept. Hence, the implementation of account would have to be changed. The "natural" solution would be to assign the code to a new association class between the two accounts but, again, current techniques for implementing association classes 
require the implementations of the participating classes to be changed because the associations are implemented via attributes.

Another problem is concerned with the handling of the synchronisation of the transfers. If the transfers are not coded in the methods of the accounts, there is no way in which the whole process can be dealt with atomically as a single transaction. Again, what is required is a mechanism via which we can superpose a layer of coordination that is separate from the computations that are performed locally in the objects and enforces the required interactions, including the synchronisation constraints. This is exactly the level of coordination that contracts provide.

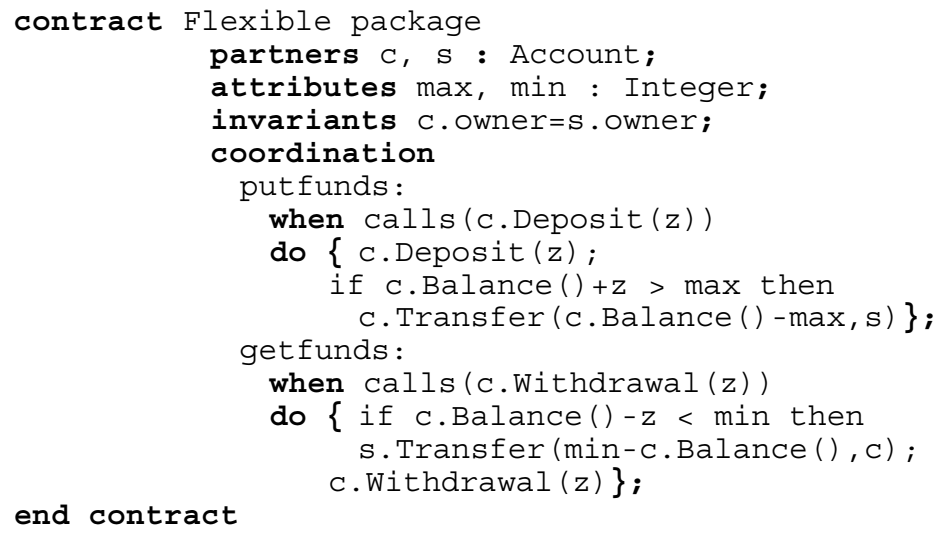

Transfers between two accounts are superposed on the operations of the partners as part of the coordination activity. Notice that, in both cases, the actions that trigger the interactions are executed as part of the synchronisation sets that define the reactions. Obviously, the execution of these actions as part of the synchronisation sets does not trigger the contract again! It may, however, trigger other contracts that the components have subscribed. For instance, the savings account may be under the coordination of a VIP-contract as well, in which case the withdrawals that are triggered by a transfer coordinated by getfunds will be handled by $v p$ as well.

\section{Semantical Aspects}

The intuitive semantics of contracts can be summarised as follows:

- Contracts are added to systems by identifying the instances of the partner classes to which they apply. These instances may belong to subclasses of the partners; for instance, in the case of the flexible package, both partners were identified as being of type account but, normally, they will be applied to two subclasses of account: a checking account and a savings account. The actual mechanism of identifying the instances that will instantiate the partners and superposing the contract is outside the scope of the paper. In Oblog, this can be achieved directly as in languages for reconfigurable distributed systems [24], or implicitly by declaring the conditions that define the set of those instances. 
- Contracts are superposed on the partners taken as black-boxes: the partners in the contract are not even aware that they are being coordinated by a third party. In a client-supplier mode of interaction, instead of interacting with a mediator that then delegates execution on the supplier, the client calls directly the supplier; however, the contract "intercepts" the call and superposes whatever forms of behaviour are prescribed; this means that it is not possible to bypass the coordination being imposed through the contract because the calls are intercepted;

- The same transparency applies to all other clients of the same supplier: no changes are required on the other interactions that involve either partner in the contract. Hence, contracts may be added, modified or deleted without any need for the partners, or their clients, to be modified as a consequence;

- The interaction clauses in a contract identify points of rendez-vous in which actions of the partners and of the contract itself are synchronised; the resulting synchronisation set is guarded by the conjunction of the guards of the actions in the set and the condition indicated in the with-clause. The execution of the synchronisation set requires the execution of all the actions in the set.

- The implementation of the synchronisation sets that are determined by the contracts can either be obtained through the primitives available in the transaction language (e.g. calls to the actions participating in the synchronisation set can be made by the regulator), or a completely new implementation can be superposed by the contract body as an alternative to the implementations available in the partners. In the latter case, the properties of the interfaces have to be preserved, namely any pre/post conditions and invariants specified through contracts in the sense of Meyer [25]. This is a useful way of bypassing legacy code as pointed out in [9]. Its implementation requires, however, that the contract body (the regulator) be awarded some sort of priority over the roles at the execution level.

- The effect of superposing a contract is cumulative: more than one contract may be active at a given state of the system. Because the superposition of a contract consists, essentially, of synchronous interactions, the different active contracts will superpose their coordinating behaviour, achieving a cumulative effect in the sense that the synchronisation set to be executed is the union of all the synchronisation sets of the active contracts and its guard is given by the conjunction of all the guards and "with" clauses associated with the active contracts. For instance, in the example of the flexible package, the transfers can also be subject to contracts that regulate the discipline of withdrawals for the particular account and client.

- In the paper, we will not address the issue of managing which contracts are active at which states. This aspect is being addressed as part of the configuration language that is being developed for assisting in the process of controlling or programming the evolution of systems. This will include logical mechanisms for reasoning about possible interactions between contracts. Preliminary work in this direction is reported in [31].

For simplicity, the examples are based on binary relationships. However, contracts may involve more than two partners. In this case, the invariant and coordination clauses may refer to all partners. In fact, contracts may be seen to correspond to syn- 
chronisation agents, as presented in the Actors model [1], that coordinate the rules of engagement of various objects participating simultaneously in the same task.

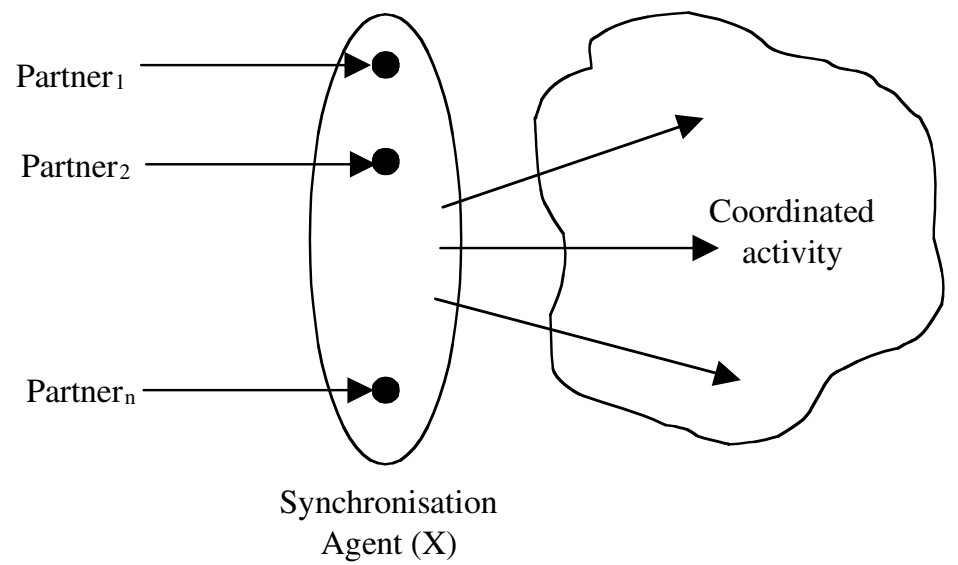

Notice that contracts in the sense of Meyer [25] fulfil a different, but complementary, role to the coordination contracts that we have described: their purpose is to support the development of object methods in the context of client-supplier relationships between objects. Therefore, they apply, essentially, to the "in-the-small" construction of systems rather than the "in-the-large" dimension, concerned with architecture and evolution, that is our target. In fact, in what concerns evolution, the use of Meyer's contracts in a non-architectural approach leads to some of the problems that we discussed in the introduction: by adopting clientship as the basic discipline for object interconnection, a bias is introduced in the way business rules get coded up, assigning to the supplier side the responsibility for accommodating changes that, from the point of view of the business rules, belong to the client. This was the case of the flexible withdrawals for VIP customers: by placing the contract on the supplier side (the account), the new rules are more easily modelled as specialisations of account whereas, in the application domain, they reflect specialisations of the client.

The intuitive semantics that we outlined above has been given both a mathematical and an implementational counterpart. The mathematical semantics draws on previous work on the categorical semantics of architectural and coordination principles. The presentation of this semantics is outside the scope of this paper. Please consult [12] for the formalisation of different kinds of superposition that lead to the identification of different kinds of contracts (regulators, monitors, etc); [13] for a formalisation of architectural connectors based on the previous formalisation of superposition, which includes the semantics of instantiation of the partners (roles); [14] for the coordination aspects as related to superposition and the application to software architectures; and $[29,30,31]$ for the application to dynamic reconfiguration, including the definition of algebraic operations on architectural connectors that are directly applicable to contracts.

The fact that a mathematical semantics exists for justifying our principle of supporting evolution through the superposition of contracts does not mean that it can be 
put into practice directly over the technology that is available today. In $[4,19]$ we have shown that, even if none of the standards for component-based software development that have emerged in the last few years (e.g. CORBA, EJB and COM) can provide a convenient and abstract way of supporting the proposed coordination principles as first-class mechanisms, an implementation can be given that is based on a design pattern that exploits some widely available properties of object-oriented programming languages such as polymorphism and subtyping. The relationship between this design pattern and the mathematical semantics is discussed in [6].

\section{Concluding Remarks}

In this paper, we have suggested that Information System Engineering can be significantly enhanced by adopting Coordination Technologies through which systems can be made more agile in responding to the turbulence that characterises current business and technological environments. More specifically, we described how a new semantic primitive - coordination contracts - can be added to current object-oriented approaches to fulfil some of the promises of Component Based Development in terms of supporting a new approach to business and technology architecture in which components can be dynamically added, modified, replaced and reconfigured $[15,20,21,28]$.

Contracts draw from several mechanisms that have been available for sometime in Software Engineering:

- Contract-based development is based on the idea of separating computation from coordination, i.e. of making clear what components in a system provide the functionalities on which services are based, and what mechanisms are responsible for coordinating the activities of those components so that the desired behaviour emerges from the interactions that are established. This idea has been promoted by researchers in the area of Programming Languages who have coined the term "Coordination Languages and Models" [e.g. 18].

- The importance of having these coordination mechanisms available as first-class entities and as units of structure in system models and designs was inspired by the role played by connectors in software architectures [2,27]. This is why we proposed contracts as a semantic primitive enriching the notion of association class.

- The ability for objects to be treated as black-box components and, hence, for contracts to be dynamically added or removed from a system without having to recompile the partners, is achieved through the mechanism of superposition (or superimposition) developed in the area of Parallel Program Design [7,10,16,22].

An effective use of the proposed coordination mechanisms further requires language and tool support for the actual run-time superposition of contracts, either in reaction to customer demands or for the enforcement of business policies. Such policies have to be understood as "invariants" that apply to "evolution time" and not "computation time". That is, they constrain the way systems can be evolved in terms of their configuration. Naturally, they will also have an impact on the computations that are performed in the system because, as we have seen, the configuration determines the 
interactions from which the global computational behaviour of the system emerges. What is important to emphasise is that decisions on which contracts can be subscribed by which classes of partners and in which states of the system are an integral part of the modelling activity. This is the direction in which we are currently working.

\section{References}

1. G.Agha, ACTORS: A Model of Concurrent Computation in Distributed Systems, MIT Press 1986.

2. R.Allen and D.Garlan, "A Formal Basis for Architectural Connectors", ACM TOSEM, 6(3), 1997, 213-249.

3. L.F.Andrade, J.Gouveia, P.Xardone and J.Camara, "Architectural Concerns in Automating Code Generation", in Proc. TC2 First Working IFIP Conference on Software Architecture, P. Donohoe (ed), Kluwer Academic Publishers.

4. L.F.Andrade and J.L.Fiadeiro, "Interconnecting Objects via Contracts", in UML'99 Beyond the Standard, R.France and B.Rumpe (eds), LNCS 1723, Springer Verlag 1999, 566-583.

5. L.F.Andrade and J.L.Fiadeiro, "Coordination: the evolutionary dimension", in Proc. TOOLS Europe 2001, Prentice-Hall, in print

6. L.F.Andrade, J.L.Fiadeiro, J.Gouveia, A.Lopes and M.Wermelinger, "Patterns for Coordination", in COORDINATION'00, G.Catalin-Roman and A.Porto (eds), LNCS 1906, Springer-Verlag 2000, 317-322

7. R.Back and R.Kurki-Suonio, "Distributed Cooperation with Action Systems", ACM TOPLAS 10(4), 1988, 513-554.

8. G.Booch, J.Rumbaugh and I.Jacobson, The Unified Modeling Language User Guide, Addison-Wesley 1998.

9. J.Bosch, "Superimposition: A Component Adaptation Technique", Information and Software Technology 1999.

10. K.Chandy and J.Misra, ParallelProgram Design - A Foundation, Addison-Wesley 1988.

11. H.Evans and P.Dickman, "Zones, Contracts and Absorbing Change: An Approach to Software Evolution", in Proc. OOPSLA'99, ACM Press 1999, 415-434.

12. J.L.Fiadeiro and T.Maibaum, "Categorical Semantics of Parallel Program Design", Science of Computer Programming 28, 1997, 111-138.

13. J.L.Fiadeiro and A.Lopes, "Semantics of Architectural Connectors", in TAPSOFT"97, LNCS 1214, Springer-Verlag 1997, 505-519.

14. J.L.Fiadeiro and A.Lopes, "Algebraic Semantics of Coordination, or what is in a signature?", in AMAST'98, A.Haeberer (ed), Springer-Verlag 1999.

15. P.Finger, "Componend-Based Frameworks for E-Commerce", Communications of the ACM 43(10), 2000, 61-66.

16. N.Francez and I.Forman, Interacting Processes, Addison-Wesley 1996.

17. E.Gamma, R.Helm, R.Johnson and J.Vlissides, Design Patterns: Elements of Reusable Object Oriented Software, Addison-Wesley 1995

18. D.Gelernter and N.Carriero, "Coordination Languages and their Significance", Communications ACM 35, 2, pp. 97-107, 1992.

19. J.Gouveia, G.Koutsoukos, L.Andrade and J.Fiadeiro, "Tool Support for Coordination Based Evolution", in Proc. TOOLS Europe 2001, Prentice-Hall, in print.

20. P.Herzum and O.Sims, Business Component Factory, Wiley 2000. 
21. J.Hopkins, "Component Primer", Communications of the ACM 43(10), 2000, 27-30.

22. S.Katz, "A Superimposition Control Construct for Distributed Systems", ACM TOPLAS 15(2), 1993, 337-356.

23. J.Kramer, "Exoskeletal Software", in Proc. 16th ICSE, 1994, 366.

24. J.Magee and J.Kramer, "Dynamic Structure in Software Architectures", in 4th Symp. on Foundations of Software Engineering, ACM Press 1996, 3-14.

25. B.Meyer,"Applying Design by Contract", IEEEComputer, Oct.1992,40-51.

26. P.Oreizy, N.Medvidovic and R.Taylor, "Architecture-based Runtime Software Evolution", in Proc. ICSE'98, IEEE Computer Science Press 1998

27. D.Perry and A.Wolf, "Foundations for the Study of Software Architectures", ACM SIGSOFT Software Engineering Notes 17(4), 1992, 40-52.

28. C. Szyperski, Component Software: Beyond Object-Oriented Programming, Addison Wesley 1998.

29. M.Wermelinger and J.L.Fiadeiro, "Connectors for Mobile Programs", IEEE Transactions on Software Engineering 24(5), 1998, 331-341.

30. M.Wermelinger and J.L.Fiadeiro, "Towards an Algebra of Architectural Connectors: a Case Study on Synchronisation for Mobility", in Proc. 9th International Workshop on Software Specification and Design, IEEE Computer Society Press 1998, 135-142.

31. M.Wermelinger and J.L.Fiadeiro, "Algebraic Software Architecture Reconfiguration", in Software Engineering - ESEC/FSE'99, LNCS 1687, Springer-Verlag 1999, 393-409. 